\title{
Linx
}

Revue des linguistes de l'université Paris X Nanterre

$53 \mid 2005$

Le semi-figement

\section{Le problème central du figement est le semi- figement}

Béatrice Lamiroy et Jean René Klein

\section{OpenEdition}

Journals

Édition électronique

URL : http://journals.openedition.org/linx/271

DOI : $10.4000 /$ linx.271

ISSN : 2118-9692

Éditeur

Presses universitaires de Paris Nanterre

\section{Édition imprimée}

Date de publication : 1 décembre 2005

Pagination : 135-154

ISSN : 0246-8743

\section{Référence électronique}

Béatrice Lamiroy et Jean René Klein, « Le problème central du figement est le semi-figement », Linx

[En ligne], 53 | 2005, mis en ligne le 14 février 2011, consulté le 20 avril 2019. URL : http://

journals.openedition.org/linx/271 ; DOI : 10.4000/linx.271

Département de Sciences du langage, Université Paris Ouest 


\title{
Le problème central du figement est le semi-figement
}

\author{
Béatrice Lamiroy - Compas-KULeuven \\ Jean René Klein-Celexrom-UCLouvain, Louvain-la-Neuve
}

\section{Introduction}

Si on consulte la littérature scientifique, francophone (e.a. Gaatone 1997, Gonzalez Rey 2002, G. Gross 1996, M. Gross 1982, 1984, 1988, Gülich \& Krafft 1997, Klein à paraître, Klein et Lamiroy 1994, 1995 et à paraitre, Lamiroy 2003 et à paraitre, Mejri 1997, 2003, Schapira 1999, Svensson 2004, Tollis 2001) ou anglophone (e.a. Everaert 1995, Hudson 1998, Hunston \& Francis 2000, Moon 1998, Nunberg et al. 1994, Tomasello 1998, Wray 2002), consacrée à la question du figement, on constate que les linguistes qui se sont penchés sur le problème sont d'accord sur un nombre de points que nous résumons ci-dessous :

$1^{\circ}$ le figement constitue un phénomène essentiel dans le fonctionnement du langage au point qu'on peut se demander s'il existe des assemblages vraiment libres. Dannell (1992: 18) considère qu'environ 30\% d'un texte est constitué d'expressions figées, ce qui correspond à l'observation de Senellart (1998) selon laquelle une phrase sur trois contient une expression figée.

$2^{\circ}$ le figement est un phénomène extrêmement complexe, polyfactoriel, ce qui explique pourquoi il échappe à toute tentative d'en fournir une définition simple et univoque. 
$3^{\circ}$ les critères du figement invoqués par les spécialistes sont multiples, mais tournent tous autour d'une série de notions centrales telles que : opacité sémantique ou non-compositionnalité du sens, expressions non motivées, sens figuratif, conventionnalité, préfabrication, restrictions syntaxiques inattendues, blocage des propriétés transformationnelles, blocage de la variation paradigmatique.

$4^{\circ}$ d'autres critères moins généraux, plus accessoires, sont parfois mentionnés, comme les traces de langue ancienne (ex. chercher noise), la non-actualisation d'un élément (ex. absence du déterminant, plier bagage), les restrictions sélectionnelles (ex. avoir avalé son parapluie) ou l'impossibilité de traduire dans une autre langue (ex. angl. to kick the bucket 'mourir' et non pas * renverser le seau) ${ }^{1}$.

$5^{\circ}$ le figement est une question de gradation : il s'ensuit qu'on parle d'expressions plus ou moins prototypiques, de ressemblance de famille et de conditions nécessaires et suffisantes (Svensson 2004 : 49) ; nous avons suggéré ailleurs (Klein \& Lamiroy à paraittre) qu'une des raisons du caractère scalaire des expressions figées est que les critères apparaissent, selon le cas, simultanément ou séparément. Ainsi, pour ne donner que cet exemple, le blocage de la transformation négative (et son contraire) caractérise un certain nombre d'expressions (1), mais pas toutes (2) :

(1) a. Les bras m'en tombent

b. *Les bras ne m'en tombent pas

c. Elle n'y va pas de main-morte

d. *Elle y va de main-morte

(2) a. Léa va abaisser ses cartes

b. Léa ne va jamais abaisser ses cartes

c. Luc nous a donné carte blanche

d. Luc ne nous a pas donné carte blanche

Par là-même il devient impossible de déterminer des conditions qui seraient 'suffisantes' de manière générale pour qu'une séquence soit étiquetée comme 'figée'.

$6^{\circ}$ la difficulté de décider du caractère figé d'une expression provient de son caractère graduel, en particulier dans la zone des figements minimaux où se situent les cas moins prototypiques: le véritable problème ne correspond pas à la limite supérieure mais à la limite inférieure du figement, c'est-à-dire non pas aux expressions fortement figées dont personne ne remet en cause le caractère idiomatique (ex. porter le chapeau, vendre la mèche), mais en revanche aux expressions peu figées ou semi-figées (ex. je vous avoue que, attirer les foudres de qqn, se dessiner à l'borizon, etc.).

\footnotetext{
${ }^{1}$ Rappelons que comme cela a souvent été remarqué, les phrases figées font partie, du point de vue de l'apprenant d'une langue étrangère, du lexique qui doit être mémorisé (Giermak-Zielinska 2000).
} 
$7^{\circ}$ le figement est à la fois une notion diachronique, comme son nom l'indique (on parle plutôt d'expressions figées que d'expressions 'fixes'), et synchronique.

Devant l'incapacité des linguistes de définir un trait commun qui serait partagé par toutes les séquences regroupées dans la catégorie des expressions figées, certains finissent par considérer l'intuition comme le meilleur critère de reconnaissance de ce qui est figé dans la langue (Misri 1987, Danell 1992, Achard \& Fiala 1997). Grunig (1997:235) avance l'idée que le trait définitoire serait en effet plutôt de type psycholinguistique que linguistique, et en particulier mémoriel : toute séquence serait susceptible d'acquérir le statut d'expression figée "à condition d'avoir un statut social solidaire d'une inscription mémorielle ». Hudson (1998: 161) abonde dans le même sens quand il dit que le seul facteur commun sous-jacent à toutes les locutions figées est d'ordre conceptuel. Moon (1998) souligne, de même, l'importance de l'institutionnalisation. Ainsi, la notion de figement serait plutôt une catégorie cognitive existant dans la mémoire des locuteurs, qu'une notion proprement linguistique. Notons que Bally (1963 : 70) avait déjà évoqué cet aspect en caractérisant les expressions figées d'une «impression de déjà vu ».

$\mathrm{Si}$ ce critère est difficile à prouver dans l'état actuel des connaissances neurolinguistiques, on peut néanmoins le tester en partie en recourant à ce qui en est le revers au niveau de la production, à savoir la prédictibilité : dans une suite mémorielle, un élément de l'ensemble suggère le tout. Misri (1987, cité par Svensson 2004 : 47) avait ainsi mis au point un test où il s'agit de remplacer les mots en schtroumpf par le mot approprié ${ }^{2}$. Les informateurs, en effet, n'avaient eu aucun problème à résoudre des phrases du genre :

(3) a. Schtroumpf-qui-peut!

b. Je vais me schtroumpfer dans un coin et piquer un petit schtroumpf

c. Schtroumpfons à la courte schtroumpf

d. Il nous la faut [la mouche] schtroumpf que schtroumpf

Toutefois, nous allons montrer dans cet article que si l'inscription mémorielle est en effet un ingrédient essentiel du figement, le nœud du problème consiste dans le fait que cette notion est elle-même une notion dynamique. Une expression peut effectivement être semi-figée parce que l'inscription mémorielle n'est pas accomplie et est en train de se mettre en place. Si Bolinger (1976) à très juste titre avait remarqué que la langue tient en équilibre entre mémoire et production - la langue est en partie automatique et en partie le produit d'un engendrement « libre » d'unités linguistiques -, le problème du figement réside dans le fait qu'on se trouve souvent à la frontière de ces deux aspects et que la séparation n'est pas étanche. Ce qui est automatique, mémorisé ou figé ne l'est en effet que "post factum», ayant été un jour engendré comme une production libre. Il suffit ainsi de remonter à des stades plus anciens de la

\footnotetext{
${ }^{2}$ En fait, l'emploi du mot schtroumpf pourrait faire croire qu'il peut figurer, en contexte, à la place de n'importe quel autre mot (comme dans la bande dessinée). Ici cependant, on pourrait substituer des points de suspension aux mots en schtroumpf et le résultat serait tout aussi prédictible :... qui peut, piquer un petit..., etc.
} 
langue pour y trouver des versions «libres » de ce qui de nos jours est figé. En effet, l'anaphore de raison dans (4a), la relative modifiant visite dans (4b) et la prédication seconde de perdre la boule en (4c) seraient actuellement inacceptables :

(4) a. Il me semble que vous avez raison; et cependant il est vrai que vous ne l'avez pas (Molière, cité Fournier 1998 : 187)

b. Le Roi alla rendre visite à Monsieur et à Madame, qui se passa fort tristement (Saint Simon, cité Fournier 1998 : 187)

c. J'avais la boule complètement perdue quand nous nous sommes retrouvés au commencement de février (Flaubert, Correspondances)

Malgré l'importance que nous accordons également à l'intuition, nous ne pensons pas, contrairement à Svensson (2004: 45), que «personne ne semble hésiter lorsqu'il s'agit de dresser des listes de différents types d'expressions figées ». Travaillant depuis environ cinq ans au recensement de toutes les expressions figées du français, y inclus celles de Belgique, du Québec et de Suisse (le projet BFQS), notre équipe (voir les noms des membres, sous 2) rencontre précisément comme principale pierre d'achoppement la difficulté de trancher au moment de retenir, ou non, telle ou telle séquence «dans la liste » des locutions figées.

L'objet de cet article étant le semi-figement, nous allons essayer de mettre le doigt sur les raisons pour lesquelles nous avons écarté une série d'expressions retenues dans la banque de données établie par M. Gross et que nous avons codées informellement PTF, c'est-à-dire "pas très figées ». Une analyse de ces cas montre, une fois de plus, que différents facteurs sont à la base de ce tri et que l'éloignement de certaines expressions de notre corpus peut être dû à des raisons fort différentes.

Nous les présenterons de façon détaillée dans la section 4. Avant cela, nous donnons un bref descriptif du projet BFQS (section 2) ainsi que des critères de figement avec lesquels nous opérons lors de la constitution de notre base de données (section 3).

\section{Les expressions verbales figées de la francophonie : le projet BFQS}

Ce projet, auquel collaborent actuellement Jean René Klein (UCLouvain), Jacques Labelle (Université du Québec à Montréal), Béatrice Lamiroy (KULeuven), Christian Leclère (CNRS, Université de Marne-la-Vallée), Annie Meunier (Université de Paris 8) et Corinne Rossari (Université de Fribourg), a été conçu à partir des travaux du « lexique-grammaire », établi au LADL (Laboratoire d'Automatique Documentaire et Linguistique) par Maurice Gross.

Ce modèle, rappelons-le, s'applique à décrire l'ensemble des types de phrases en fonction des items lexicaux qui conditionnent leur syntaxe (Lamiroy 1998). Cette vaste recherche, consacrée au départ à la classification des constructions françaises à verbe simple (Boons, Guillet \& Leclère 1976, Gross 1975, Guillet \& Leclère 1992), a été complétée depuis les années 80 par l'étude de milliers de combinaisons verbales considérées comme figées (M. Gross 1982, 1988). L'ensemble des expressions répertoriées par M. Gross appartient au «français de France». 
Le projet B(elgique)F(France)Q(Québec)S(Suisse) propose d'élargir la description à une grande partie de la francophonie ${ }^{3}$. Les variantes géographiques du français, le plus souvent décrites de façon sporadique tant dans les dictionnaires que dans les ouvrages spécialisés, font ici l'objet d'un inventaire et d'un classement systématiques qui s'inspirent du modèle conçu au départ pour les expressions appartenant au français hexagonal.

Pour recenser les expressions, nous avons suivi une double démarche. Nous avons commencé par séparer, à partir de la liste d'environ 45000 expressions figées établie par M. Gross, les expressions communes à toutes les variétés (notées BQFS). La grande majorité des expressions sont en effet connues à travers les quatre variétés et constituent le véritable tronc commun des expressions figées du français. Les estimations faites à partir du travail réalisé jusqu'à maintenant laissent supposer qu'il s'agit de 75 à 80\% des expressions. En voici quelques exemples :

(5) a. Luc est au septième ciel

b. Max est allé un peu vite en besogne

c. Ce sac $m^{\prime}$ a coûté les yeux de la tête

d. Le travail lui casse les pieds

e. Va au diable!

Dans la même liste de départ, nous avons identifié les expressions qui ressortissent soit au français de France uniquement soit à plusieurs variétés, sans être communes à toutes, par ex.

(6) a. (F) Ces gens-là pètent dans la soie

b. (F) Tu es tombé du lit ce matin!

c. (BFS) Il l'aurait bouffé tout cru

d. (FQ) Léa a attrapé mal

e. (FS) Va te faire lanlaire! 'être très riche'

'se lever très tôt'

's'adresser à qq d'un air menaçant'

'attraper un refroidissement'

'se faire rabrouer'

Dans l'état actuel des travaux, tout le corpus a été trié et codé en fonction des quatre variétés. L'élaboration de la lettre A a permis de mettre au point la méthodologie très complexe de la description et d'établir un protocole qui devra être appliqué au reste de l'alphabet. Le protocole élaboré est le résultat d'un travail considérable portant sur la question du figement (c'est-à-dire le tri des expressions, cf. ci-dessous $\int 4$ ), l'analyse syntaxique des expressions, l'harmonisation des définitions, la recherche d'exemples significatifs ainsi que l'établissement des expressions synonymes appartenant à des variétés géographiques différentes.

La description du sous-ensemble F, n'ayant à notre connaissance jamais été entreprise auparavant, constitue sans doute un des résultats les plus originaux du projet et montre qu'il ne faut pas confondre, comme on le fait souvent, français commun et français de France. En outre, la connaissance précise des diverses intersections des variétés géographiques respectives correspond également à un aspect innovateur de ce projet.

\footnotetext{
${ }^{3}$ Le travail n'inclut pas le français d'Afrique ni celui des DOM-TOM.
} 
Dans un deuxième moment, les équipes belge, québécoise et suisse ont établi chacune une liste aussi complète que possible des expressions propres à leur communauté linguistique. Toutes sortes de sources ont été exploitées: répertoires publiés (par ex. Bal et al. 1994, Massion 1987 pour les belgicismes, Meney 1999 pour les québecismes, Thibault \& Knecht 1997 pour les helvétismes), journaux, littérature régionale, langage parlé, moteurs de recherche (Google), etc.

Ainsi on trouve pour les expressions communes coûter les yeux de la tête ou aller au diable un équivalent dans chacune des variétés :

(7) a. Cette voiture coûte les yeux de la tête (BFQS)

b. Cette voiture coûte un os/pont (B)

c. Cette voiture coûte bonbon $(\mathrm{F})$

d. Cette voiture coûte un bras (Q)

e. Cette voiture coûte le lard du chat/ un saladier $(\mathrm{S})$

(8) a. Va au diable! (BFQS)

b. Va te faire enrager!

c. Va te faire lanlaire! (FS)

d. Va chez le bonhomme! (Q)

Les données rassemblées ont donc fourni des belgicismes, des francismes, des helvétismes et des québecismes qui ont été ajoutés au corpus initial. Ces expressions ont ensuite été comparées systématiquement, chaque équipe portant un jugement sur la présence de ces expressions dans son propre domaine géographique. Ce travail fournit de nouvelles intersections, par ex.

(9) a. attendre de midi à quatorze heures (QS)

'attendre longtemps'

b. avoir encore qqc de bon (BS)

'avoir encore droit à qqc'

Il va de soi que les jugements, bien que fondés sur l'intuition des linguistes ainsi que sur une base documentaire constituant un important moyen de vérification, n'ont pas de valeur absolue, pour plusieurs raisons. Premièrement parce que la notion même de variante diatopique couvre en fait plusieurs sous-types, comme Goosse (1977) l'a démontré pour les belgicismes. En outre, même de solides enquêtes sociolinguistiques ne pourraient rendre compte de la variation en fonction de l'âge, l'appartenance sociale, le degré d'instruction, le milieu de travail, etc. des locuteurs. Et enfin, nous sommes tout à fait conscients qu'à l'intérieur d'un même domaine de la francophonie, de multiples variations régionales internes coexistent. Pour certains domaines comme la France et la Suisse, elles ont été recensées dans les excellents ouvrages de Rézeau (2001) et de Thibault et Knecht (1997) respectivement. Ainsi, une expression telle que avoir facile/ difficile (Goosse 1992), bien que caractéristique du français de Belgique, est connue aussi en France, surtout en Lorraine et dans le Nord.

Notons que la variation notée pour chacune des expressions porte non seulement sur la variation lexicale mais aussi sur des aspects mineurs. En effet, on observe parfois une légère différence quant à la préposition (7), au nombre (8) ou au déterminant (9) : 
(10) a. travailler an noir (BFQS)

b. travailler en noir (B)

(11) a. annoncer la couleur (BFS)

'dévoiler ses intentions'

b. annoncer les/ses couleurs $(\mathrm{Q})$

(12) a. attendre famille (B)

b. attendre la famille (Q)

c. attendre de la famille $(\mathrm{S})$

Le schéma qui suit, réalisé par C. Leclère (Lamiroy et al. 2003), résume les différents cas de figure liés à la variation diatopique. Soit l'expression est commune et donc BFQS, soit elle n'appartient qu'à un des domaines (B, F, Q ou $\mathrm{S}$ ) ou à plusieurs d'entre eux (par ex., BF, BFQ, QS, etc.).

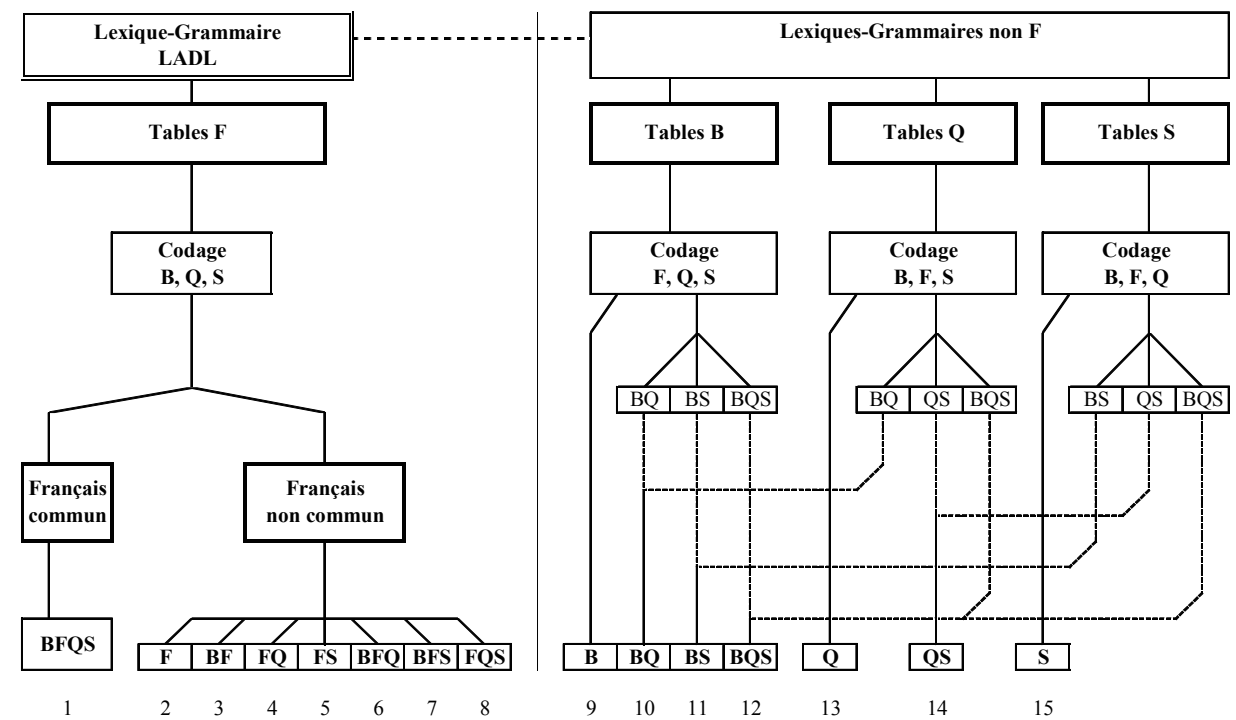

Du point de vue théorique, le but du projet BFQS est donc double : d'une part constituer un véritable thesaurus des expressions figées du français et d'autre part, rendre compte de la variation à travers la francophonie, en Belgique, en France, au Québec et en Suisse. Par ailleurs, grâce à une analyse syntaxique systématique de toutes les expressions recensées, une série de produits dérivés deviennent envisageables à partir des résultats descriptifs obtenus, allant de manuels pour allophones aux outils servant à la traduction automatique d'une variété géographique à une autre ou à la neutralisation de textes régionaux par transposition en français 'commun'.

\section{La notion d'expression figée}

Ce dictionnaire consacré aux expressions verbales figées dans quatre domaines de la francophonie repose bien entendu sur la notion, à la fois cruciale et extrêmement difficile à définir, du figement. 
Nous développons brièvement ci-dessous le point de vue adopté dans le projet $\mathrm{BFQS}$ par rapport à cette question.

Précisons d'emblée que le figement phraséologique envisagé ici est lié à la polylexicalité, soit la présence de plus d'une unité lexicale ou «mot », sans quoi tous les phénomènes de lexicalisation tels que les noms composés, les métaphores lexicalisées (ex. étoile pour 'vedette') pourraient être assimilés à des figements. Par exemple nous n'avons pas retenu les cas illustrés en (13a-b) :

(13) a. Purée!

b. On l'a jeté dans un cul-de-basse-fosse

parce que le premier est un mot simple et que cul-de-basse-fosse est un nom composé figé qui ne constitue pas une expression verbale avec le verbe jeter: il peut apparaître, dans le même sens, avec toutes sortes de verbes et dans toutes sortes de positions syntaxiques, ex. Ce cul-de-basse-fosse date du Moyen-Age, On a visité le cul-de-basse-fosse de ce vieux château, etc.

Dans le cas des expressions verbales figées que nous avons retenues, ce qui constitue la principale différence avec la syntaxe des verbes simples, est qu'un argument au moins est figé, au sens où il est irremplaçable ou fait partie d'un choix restreint, en plus du verbe :

(14) a. le sujet: les dés sont jetés 'il n'y a plus que le sort qui décide'

b. l'objet : prendre la plume 'écrire'

c. un complément prépositionnel: cracher au bassinet 'payer ce qu'on doit'

d. un complément adverbial : $y$ aller gaiement

De ce fait, les contraintes dues à la seule valence verbale sont exclues du domaine des expressions figées (Lamiroy 2003).

Intuitivement, dans une séquence figée, tout usager perçoit aisément l'opacité sémantique. En effet, très souvent, le sens n'est pas le produit de la somme des sens des éléments lexicaux individuels: on a beau connaittre le sens de avaler et celui de coulewvre, on n'atteint pas le sens 'supporter des affronts sans rien dire'. Il en va de même pour le belgicisme aller à la moutarde qui veut dire 'se faire rabrouer', l'helvétisme aller sur Soleure qui a le sens de 'se soûler', ou pour le québecisme attendre les samvages signifiant 'être enceinte'. A ce titre-là, ces unités polylexicales fonctionnent en effet comme les mots simples : ce sont des dénominations (Kleiber 1989) dont le sens est figé par convention.

Ce critère vaut, a priori en quelque sorte, pour les locutions qui contiennent des mots fossiles qui n'apparaissent plus qu'à l'intérieur de l'expression. Le cas n'est pas inusuel, peut-être moins qu'on ne le pense : en voici quelques exemples, de type verbal (10a) ou adverbial (10b) :

(15) a. avoir maille à partir, y avoir maldonne, sans coup férir, battre la chamade, chercher noise, rester coi, hocher la tête, conter fleurette, se mettre martel en tête, aller à vau-l'eau, en savoir gré à qqn, faire la nique à qqn, prendre la poudre d'escampette, briller comme des 
Le problème central du figement est le semi-figement

escarboucles, tomber dans l'escarcelle, prêter main-forte à qqn, avoir la berlue, damer le pion à qqn, la bailler belle, etc.

b. à la sauvette, à la bonne franquette, à contrecoeur, à la queue leu leu, au gré de, d'emblée, au fur et à mesure, il y a belle lurette, à tire-larigot, à l'improviste, tout de go, à bon escient, sur ces entrefaites, en guise de, en tapinois, etc.

Ce critère, suffisant peut-être pour le locuteur natif et 'naï', ne suffit toutefois pas pour le linguiste. Pas mal de travaux, dont ceux cités plus haut, ont ainsi proposé une critériologie du figement fondée à la fois sur la syntaxe, le lexique et la sémantique, sans définir ce qui est hiérarchiquement dominant. En effet, non seulement le sens d'une expression est non compositionnel, mais les éléments constitutifs de l'expression subissent également un figement formel. Celui-ci se révèle le plus clairement par deux propriétés, l'une lexicale et l'autre morphosyntaxique. En effet, les expressions entrent dans des paradigmes limités où la variation lexicale habituelle de la syntaxe libre fait défaut :

(16) a. prendre ses jambes à son cou

b. *prendre ses pieds à sa nuque

c. *prendre les jambes au cou

(17) a. ne pas bouger le petit doigt

b. *ne pas bouger l'index

c. ${ }^{*}$ ne pas plier le petit doigt

Et d'autre part ils sont sujets à des contraintes de type morphosyntaxique :

(18) a. porter le chapeau vs * porter un/ce chapeau ${ }^{4}$

b. il a vendu la mèche vs *la mèche a été vendue

c. les carottes sont cuites vs *les carottes qui ont été cuites

Un point sur lequel on ne peut insister suffisamment est que nous considérons la question du figement comme une notion foncièrement graduelle. En effet, lorsqu'on applique les critères usuels à des ensembles très vastes d'expressions comme nous le faisons dans le projet BFQS, on s'aperçoit qu'ils manifestent des degrés variables de pertinence. Le problème se pose particulièrement pour des expressions interprétables analytiquement, mais sujettes à des restrictions paradigmatiques ou à des contraintes morphosyntaxiques. Ainsi, le sens d'une expression comme garder sous le coude est plus aisément accessible que celui de avaler des couleuvres, outre que certaines variantes verbales sont possibles, telles rester ou tenir sous le coude. L'expression est donc moins figée que avaler des coulewvres dont le sens est opaque et qui ne connait pas de variante verbale. La même observation s'applique évidemment aux expressions qui n'appartiennent pas au français commun, par ex. parmi les belgicismes, faire de son nez 'protester' est sans doute ressenti comme plus figé que faire le fou avec qqn 'se moquer de qqn'. Si une représentation scalaire permet sûrement de mieux rendre compte des

${ }^{4}$ Ces séquences sont bien sûr acceptables au sens littéral. 
phénomènes de figement, la difficulté de trancher se situe dans la zone des figements minimaux, où se situent les cas moins "prototypiques » et au-delà de laquelle on quitte la zone de la syntaxe figée. Les cas les plus compliqués sont des expressions où le figement est moins spectaculaire parce que les critères ne se manifestent pas tous à la fois. Il arrive effectivement assez souvent que le sens soit relativement transparent, mais que des contraintes lexicales et/ou morphosyntaxiques soient présentes, par ex. arriver à bon port a été retenu dans le corpus BFQS comme expression malgré son sens relativement analytique à cause de la limitation paradigmatique *arriver à manvais port, ou aller de soi, à cause de la contrainte morphosyntaxique *aller de lui. De même, des séquences apparemment libres telles que aller à l'école ont été retenues parce que l'interprétation locative, littérale n'est pas la seule possible. Dans il n'est pas allé à l'école, la séquence peut signifier 'il n'a reçu aucune instruction'. A ce stade très ténu du figement, un corpus d'expressions verbales figées prendra donc des proportions très variables, selon que l'on prendra en compte certains indices à première vue peu spectaculaires de figement ou non.

Poussant le raisonnement jusqu'au bout, on pourrait aller jusqu'à considérer la syntaxe libre comme un degré minimal de figement puisque, si l'on tient compte des traits de sélection, pratiquement aucun verbe ne se construit avec n'importe quel argument. Or les contraintes dues à la seule valence verbale sont bien sûr exclues du domaine des expressions figées. Un cas limite entre figement et valence est celui des "classes d'objets", notion proposée par G. Gross (1996), pour désigner des paradigmes qui rassemblent les objets possibles ressortissant à une catégorie sémantique identifiable. Ainsi, un verbe tel que prendre prend un sens différent selon qu'il se combine avec un des paradigmes suivants :

(19) a. prendre un train/ un avion/ un bus, etc. 'utiliser un moyen de transport'

b. prendre un verre/ un repas/ un apéritif, etc. 'ingérer, absorber'

c. prendre un chemin/ une route/ une rue, etc. 's'engager dans une voie'

Comme il s'agit de classes limitées, nous avons retenu ce genre d'expressions verbales dans notre corpus BFQS, par ex.

(20) a. apprendre pour médecin/infirmière/institutrice... (B)

b. ne pas venir à $\mathrm{x}$ francs/minutes/jours...

En résumant, nous avons retenu comme expression verbale figée trois types majeurs d'expressions.

$1^{\circ}$ Celui des expressions non compositionnelles, franchement opaques du point de vue sémantique :

(21) a. adorer le vean d'or (BFQS)

b. faire des ruses à qqn (B)

c. ambitionner sur le pain bénit $(\mathrm{Q})$

d. être l'heure de police (S)

e. danser devant le buffet $(\mathrm{F})$ 'vénérer l'argent'

'causer des ennuis'

'abuser d'une situation'

'être l'heure d'aller se coucher'

'avoir faim' 
Il est évident que l'opacité sémantique va de pair avec des contraintes d'ordre lexical et morphosyntaxique :

(22) a. *adorer la vache d'or

b. *faire une ruse à qqn

c. *danser devant ce buffet

$2^{\circ}$ Un deuxième type concerne des expressions qui ne manifestent pas nécessairement d'opacité sémantique, mais sont contraintes du point de vue morphosyntaxique. On entend par-là des restrictions soit au niveau des transformations syntaxiques soit au niveau des alternances morphologiques. Ainsi prendre la fuite (BFQS) manifeste, malgré sa transparence, un haut degré de figement syntaxique (Martin 1997):

(23) a. *c'est la fuite qu'il a prise

b. *la fuite a été prise

c. *qu'est ce qu'il a pris ? la fuite

Une contrainte morphosyntaxique peut justifier également qu'on retienne une expression comme locution figée, sans qu'il y ait nécessairement opacité sémantique, par ex.

(24) a. prendre ses désirs pour des réalités (BFQS)

b. *prendre des désirs pour de la réalité

(25) a. faire des chatouilles (B) 'chatouiller'

b. *faire une chatouille

(26) a. aller en commissions ( $\mathrm{S}$ ) 'aller faire les achats quotidiens'

b. *aller à commission

$3^{\circ}$ Pour certaines expressions intuitivement transparentes et qu'on pourrait caractériser de peu figées à première vue, nous avons considéré que le figement est cependant présent: seulement, il ne se caractérise que par des limitations paradigmatiques de certains arguments. L'absence de variation paradigmatique peut affecter toutes les fonctions syntaxiques, qu'il s'agisse de sujet (27), du complément d'objet (28), d'un complément adverbial (29), etc. :

(27) a. le vent chasse (B) 'il y a un courant d'air'

b. *l'air chasse

(28) a. prendre son mal en patience (BFQS)

b. *prendre sa souffrance en patience

(29) a. $y$ aller gaiement (BFQS)

b. *y aller tristement

Au-delà de ce stade se trouvent les constructions libres, qui, rappelons-le, impliquent toutefois, elles aussi, un minimum de contrainte, tant sur le plan sémantique que sur le plan formel. En effet, même les phrases dites libres possèdent 
toujours un certain degré de contrainte ne fût-ce que par le fait qu'elles sont conditionnées par leur valence (Lamiroy 2003). Ainsi, on ne pourra jamais manger que ce qui est mangeable, prendre ce qui est " prenable », etc. : manger un train en chocolat vs *manger un train (Lamiroy 2003).

Il est intéressant de souligner que les expressions figées, contrairement à ce qu'on pourrait croire, abondent dans la langue technique, comme le montrent les quelques exemples donnés ci-dessous 5 . Nous les avons écartées de notre base de données parce que le dictionnaire que nous envisageons est consacré à la langue générale.

(30) a. langue des sports et des jeux

remporter aux points [boxe], faire le breack. [tennis], virer au cabestan, remonter au vent [voile], se recevoir sur une jambe/main/un pied [athlétisme], pêcher au gros [pêche], rien ne va plus [roulette]

b. langue juridique arrêter au nom de la loi, lever la grosse, passer en simple police, intenter une action en justice, appeler à la barre des témoins

c. téléphonie appeler à frais virés, appeler à charges renversées

d. secteur automobile aligner les roues

e. langue de l'administration radier du rôle de $\mathrm{N}$, présenter à la signature de $\mathrm{N}$

f. langage militaire verser dans la réserve

g. langue littéraire en accepter l'angure, recevoir sous la Coupole

h. langue de l'économie atteindre son plafond/son plancher etc.

Il va de soi que les variantes géographiques du français peuvent présenter le même genre d'expressions, ainsi on trouve en (B) faire la géométrie, qui signifie 'aligner les roues d'une voiture', ou en $(\mathrm{F})$ rouler sur trois pattes 'rouler sur trois cylindres'.

En conclusion, notre position au sein du projet BFQS tend à retenir un maximum de locutions verbales figées, puisque nous accueillons toute une série de séquences contraintes du point de vue paradigmatique et/ou (morpho)syntaxique. Autrement dit, nous étendons, à la suite de M. Gross, la notion de figement au-delà de la seule non-compositionnalité sémantique. D'autre part, nous excluons, tout comme

${ }^{5}$ De même qu'il est illusoire de croire que la langue technique échappe à la polysémie. 
Maurice Gross, les contraintes dues à la valence, sauf pour certaines classes d'objet qui se trouvent à la limite entre la valence et le domaine du figement.

Toutefois, nous avons écarté toute une série d'expressions verbales semi-figées ou figées en apparence qui figurent dans la base de données originale de M. Gross pour des raisons diverses que nous allons détailler ci-dessous. Ainsi, notre position, tout en étant assez large dans la mesure où nous refusons de nous limiter à l'opacité idiomatique comme seul critère de figement, est malgré tout moins maximaliste que celle de M. Gross.

\section{Le semi-figement}

Parmi les expressions que nous avons écartées, on peut distinguer plusieurs sous-types, de nature assez diverse d'ailleurs. Le premier englobe les cas de type pragmatique (4.1), le deuxième regroupe des expressions que nous avons refusées parce qu'elles représentent des variantes verbales peu naturelles des véritables expressions. Un troisième groupe (4.3) correspond à des éléments figés qui sont des expressions verbales en apparence seulement: en fait les structures contiennent des adverbes figés (composés). Un quatrième type réunit tous les cas où on peut croire à première vue à des variétés figées de type géographique, alors qu'il s'agit en fait de structures contenant des unités lexicales seulement (4.4). Le dernier type enfin, représente véritablement des cas de semi-figement, bien que ceux-ci puissent encore appartenir à deux catégories distinctes, à savoir les collocations, d'une part, et les emplois métaphoriques, d'autre part (4.5).

\subsection{Expressions de type "pragmatique »}

Il s'agit de combinaisons figurant dans la base de données de M. Gross, dont le sens est compositionnel et la morphosyntaxe libre, mais qui entraînent une inférence pragmatique particulière, par ex.
a. prendre un pull
'prévoir qu'il fera froid'
b. prendre un parapluie
'prévoir une menace de pluie'

Nous n'avons pas retenu ce type de séquences, car rien ne permet de prédire si leur sens inférentiel se lexicalisera un jour ou non.

\subsection{Les variantes verbales}

Une deuxième catégorie regroupe les cas des variantes «forcées» des expressions. Si le propre de beaucoup d'expressions est de disposer de variantes, que nous indiquons sous l'entrée même de l'expression, certaines variantes nous ont semblé artificielles ou peu courantes. Ces séquences, retenues dans la base de données de M. Gross, ont été codées EXTAB (" extensions abusives») et ont été écartées. La variation peut concerner le verbe de l'entrée ou un des arguments, nominal ou prépositionnel. Nous donnons en (32) des exemples de variantes qui ont été retenues parce qu'elles nous ont semblé naturelles, tandis que (33) illustre des cas de pseudovariantes qui, elles, n'ont pas été retenues. Il est évident que la frontière entre les deux 
est parfois délicate à tracer, les variantes étant forcément sujettes à des fluctuations d'usage :

(32) a. accrocher une casserole à la queue de qqn
var. attacher
var. aller
var. arranger
var. en arriver
var. se magner
var. entendre
var. ?? repos
var. ?? attraper
var. ?? demeurer
var. ?? les lèvres/les doigts
var. ?? regarder
var. ?? lire derrière les mots
var. ?? mettre

b. marcher sur les brisées de qqn

c. abîmer le portrait à qqn

d. en venir au fait

e. s'activer le cul

f. en apprendre de(s) belles/bonnes sur

(33) a. perdre le sommeil

b. prendre le taureau par les cornes

c. cela reste à voir

d. se pourlécher les babines

e. voir $q 9$ à l'cuvre

f. lire entre les lignes

g. faire obstacle

Notons que le phénomène des variantes se produit également pour des expressions appartenant au français non commun, par ex.

(34) a. s'asseoir sur son steack (Q) 'ne plus rien entreprendre'

var. rester

b. arriver comme des figues après Pâques (B) 'arriver trop tard'

var. venir

\subsection{Expressions figées en apparence}

Certaines expressions qui à première vue peuvent sembler figées ne le sont pas quand on les analyse de plus près : en effet, il s'agit non pas d'expressions verbales mais d'adverbes figés. La nature figée de l'adverbe peut créer l'illusion qu'on a affaire à une locution verbale, alors qu'en réalité le verbe est variable, par ex. ${ }^{6}$

(35) a. se montrer sous son vrai jour

b. appeler de toute son âme

c. s'arrêter pile

d. arriver à brûle-pourpoint

e. atteindre de plein fouet

f. ne pas avancer d'un poil

g. arriver à point nommé

h. travailler pour des clous/prunes/ haricots/nèfles aussi : apparaître, se présenter, etc.

aussi : désirer, soubaiter, etc.

aussi : tomber, arriver, etc.

aussi : venir, se présenter, demander, etc. aussi : heurter, frapper, etc. aussi : bouger, reculer, etc. aussi : survenir, intervenir, etc. aussi : faire qqc, se fatiguer, etc.

\footnotetext{
${ }^{6}$ La même observation vaut évidemment pour des $\mathrm{N}$ composés, par ex. jeter dans un cul-de-basse-fosse n'est pas retenu parce qu'on trouve également Ce cul-de-basse-fosse date du Moyen Age, visiter le cul-de-bassefosse du château, etc.
} 
i. coûter dans ces eaux-là

j. avancer à vue d'cil aussi : valoir, se situer, etc.

aussi : vieillir, grossir, maigrir, etc.

Cette observation vaut évidemment également pour toutes les variantes du français. Ainsi partir en stoemelings (B), signifiant 'partir à l'anglaise' n'a pas été retenue en tant qu'expression parce que c'est le complément adverbial qui est figé, et non la locution entière (cf. arriver en stoemelings, faire qqc en stoemelings, s'approprier qqc en stoemelings, etc.)

\subsection{Variétés régionales}

Une autre catégorie encore, particulière au recensement des variantes géographiques du français, correspond à des séquences contenant un élément à spécificité géographique et donc opaques aux yeux du locuteur du français commun. Or, ici également, la combinaison d'un tel élément avec certains verbes peut faire croire qu'on se trouve devant une expression figée, alors qu'il s'agit d'une variation purement lexicale (Klein \& Rossari 2003). Ainsi, la série suivante prend les apparences d'un paradigme « figé » à variation diatopique. Or ce ne sont que les substantifs qui ont un sens particulier parce que régional :
a. boire un péquet $(\mathrm{B})$
'boire un alcool fort'
b. boire une petite bleue $(\mathrm{S})$
'boire un alcool fort'
c. boire une verte (F)
'boire un alcool fort'
d. boire une liqueur $(\mathrm{Q})$
'boire une boisson gazeuse'

Il en va de même pour des séquences telles que
a. éteindre/ allumer le robinet
b. prendre un dix heures (BS)
c. peser sur un bouton $(\mathrm{S})$

'fermer/ouvrir'

'prendre une collation à dix heures du matin'

'appuyer sur un bouton'

\subsection{Expressions semi-figées}

De la même façon qu'il y a deux aspects constitutifs de la nature figée d'une expression (formel et sémantique), les facteurs à la base de ce qu'on pourrait considérer comme des séquences semi-figées seraient également de double nature. C'est précisément en examinant de plus près ces locutions quasi ou presque figées qu'on se rend compte des mécanismes qui sont à l'origine du processus du figement. Il se trouve qu'il y en a deux essentiellement, de nature fondamentalement différente. D'une part, il y aurait la tendance universelle pour certains éléments de la langue à apparaitre volontiers ensemble, au point où ces combinaisons peuvent finir par être institutionnalisées : c'est le cas des collocations, associations préférentielles mais non contraignantes. Le figement, qui résulte d'une fréquence d'emploi, concerne avant tout la forme, sans affecter nécessairement le sens des éléments constituants, par ex.

(38) a. accepter avec plaisir

b. abuser du temps/de la bonté/de la gentillesse de qqn 
c. n'avoir rien à ajouter/dire de plus

d. ça va passer

e. attendre son tour/sa chance

f. avancer sur son temps/âge

g. ça tient à peu de chose

h. on n'aurait jamais cru/dit/pensé/imaginé ça

i. ça appelle une remarque

D'autre part, il y a un mécanisme d'ordre sémantique, mais tout aussi général, à savoir l'exploitation de la polysémie des mots, et en particulier le recours à la métaphore. Or comme d'autres l'ont souligné (Svensson 2004 : 140), la métaphore à elle seule ne garantit pas le figement: les œuvres littéraires (la poésie en particulier) en abondent, sans qu'on attribue à ces séquences le statut d'expressions figées :

(39) a. Les sanglots longs des violons de l'automne

blessent mon cœur d'une langueur monotone (Verlaine, Chanson d'automne)

b. Je suis un cimetière abhorré de la lune

(...) Je suis un vieux boudoir plein de roses fanées (Baudelaire, Spleen)

L'exemple des textes littéraires montre bien à quel point la «locutionalité » (Achard \& Fiala 1997) est conditionnée par l'institutionalisation de la séquence. La tendance (et la tentation) à considérer quelque chose comme figé sera d'autant plus grande que les deux facteurs sont en place simultanément. Or l'un et l'autre ne suffisent pas à eux seuls à conférer à une suite de mots, le statut d'expression figée. Cidessous figurent des exemples de locutions verbales contenant une métaphore (verbale ou nominale) qui apparaissent dans la base de données de M. Gross et que nous n'avons pourtant pas retenues sur la base du principe que métaphore ne vaut pas figement, du moins pas nécessairement. Bien que les métaphores illustrées ci-dessous apparaissent souvent dans les combinaisons données, celles-ci ne sont pas exclusives :

(40) a. attirer les foudres de qqn (aussi : susciter, provoquer)

b. mijoter dans son jus (aussi : crever, mariner)

c. atteindre des sommets, le summum (aussi : arriver à)

d. éclater au grand jour (aussi : à la lumière du jour)

e. se dessiner à l'horizon (aussi : se profiler)

f. ne pas s'arrêter en si bon chemin (aussi : en si beau chemin)

g. acheter le silence de qqn (aussi : le vote de qqn)

h. s'aérer les idées (aussi : se changer)

i. accompagner dans la tombe (aussi : dans l'autre monde, dans son dernier voyage)

j. s'attendre à des miracles (aussi : à des merveilles) 
Le problème central du figement est le semi-figement

\section{Conclusions}

Comme nous l'avons suggéré au début de l'article, l'expérience du projet BFQS qui nous amène à prendre en considération un très grand nombre de locutions, allant du très figé à du très peu figé, nous a confrontés au problème extrêmement complexe de la définition de ce que c'est qu'une expression figée. La difficulté du problème tient essentiellement à deux facteurs : d'une part le fait que le phénomène du figement est polyfactoriel et, partant, scalaire, dans la mesure où tous les traits définitoires ne se présentent pas toujours simultanément et d'autre part, le fait qu'il s'agit d'un processus foncièrement dynamique. Comme tout phénomène de type diachronique qu'on veut appréhender, l'éclairage qu'on peut y apporter est fatalement une « lumière qu'on porte sur le dos»(Toynbee), c'est-à-dire qu'une définition n'est possible que lorsque le processus est accompli. Autrement dit, le semi-figement est bien le problème central du figement parce que tous les cas où un figement potentiel est en cours, que ce soient pour des raisons formelles ou sémantiques, échappent provisoirement à une catégorisation définitive. Cela dit, on peut essayer, comme nous l'avons fait ici, de mettre le doigt sur un certain nombre de mécanismes divers qui jouent chacun un rôle dans ce processus, très complexe mais fascinant, du figement.

\section{RÉFÉRENCES}

ACHARD, P. \& FiALA, P. (1997), La locutionalité à géométrie variable, in Fiala, P., Lafon, P. \& Piguet, M.F. (éds), pp. 273-284.

Bal, W., Doppagne, A., Goosse, A., Hanse, J., Lenoble-Pinson, M., Pohl, J. \& Warnant, L. (1994), Belgicismes. Louvain-la-Neuve : Duculot.

BALly, C. (1962), Traité de Stylistique française. Genève : Librairie de l'Université Georg \& Cie.

Bolinger, D. (1977), Meaning and Memory. Forum Linguisticum, 1, pp. 1-14.

BoOns, J.-P., Guillet, A. \& LeCLÈRE, C. (1976), La structure des phrases simples en français. Les constructions intransitives. Genève : Droz.

DAnNelL, K. J. (1992), Nothing but phrases. About the distribution of idioms and stock phrases, in Edlund, L.E. \& Persson, G. (éds), Language : the time machine. Umeå : Umeå University.

EveraerT, M. et al. (éds) (1995), Idioms. Hillsdale : N.J., Erlbaum.

Fiala, P., LAFOn, P. \& Piguet, M.F. (éds) (1997), La locution : entre lexique, syntaxe et pragmatique. INALF, coll. St-Cloud. Paris : Klincksieck.

FOURNIER, N. (1998), Grammaire du français classique. Paris : Belin.

GAATONE, D. (1997), La locution : analyse interne et analyse globale, in Martins-Baltar M. (éd.), pp. 165-177.

GibBs, R. (1995), Idiomaticity and Human Cognition, in EveraerT, M. et al. (éds), pp. 97-117. 
Béatrice Lamiroy et Jean René Klein

Giermak-Zielinska, T. (2000), Les expressions figées. Propositions pour un traitement contrastif. Varsovie : Publications de l'Institut de Philologie Romane.

GuilLET, A. \& LECLÈRE, C. (1992), La structure des phrases simples en français. Les constructions locatives. Genève : Droz.

GonZalez Rey, I. (2002), La phraséologie du français. Toulouse : Presses de l'Université du Mirail.

Goosse, A. (1977), Qu'est-ce qu'un belgicisme? Bulletin de l'Académie Royale de langue et de littérature françaises de Belgique, LV, pp. 345-367.

Goosse, A. (1992), Le dossier d'avoir facile in Goosse, A. (éd.), Mélanges de grammaire et de lexicologie françaises. Louvain-la-Neuve : Peeters, pp. 324-351.

Gross, G. (1996), Les expressions figées en francais. Noms composés et autres locutions. Paris : Ophrys.

Gross, M. (1975), Méthodes en Syntaxe. Paris : Hermann.

Gross, M. (1982), Une classification des phrases «figées » du français, Revue Québecoise de Linguistique, 11.2, pp. 151-185.

Gross, M. (1984), Une classification des phrases «figées» du français, in Attal, P. \& Müller, C. (éds), De la Syntaxe à la Pragmatique. Amsterdam : Benajamins, pp. 141-180.

Gross M. (1988), Les limites de la phrase figée, Langages, 90, pp. 7-22.

GRUNIG, B-N. (1997), La locution comme défi aux théories linguistiques : une solution d'ordre mémoriel ? in Martins-Baltar, M. (éd.), pp. 225-240.

GuELICH, E. \& KRAFFT, U. (1997), Le rôle du préfabriqué dans les processus de la production discursive, in Martins-Baltar, M. (éd.), pp. 241-276.

Hudson, J. (1998), Perspectives on fixedness : applied and theoretical. Lund Studies in English, 94. Lund, Lund University Press.

Hunston, S. \& Francis, G. (2000), Pattern Grammar. Amsterdam : Benjamins.

Kleiber, G. (1989), Sur la définition des proverbes, in Greciano, G. (éd.), Phraséologie contrastive. Strasbourg : Université des Sciences Humaines, pp. 233-253.

KLEIN, J.R. à paraitre. Le figement, un concept aussi essentiel que fluent. Réflexions à travers la synchronie et la diatopie, in Actes du Colloque EUROPHRAS, Bâle, août 2004, sous presse.

KLein, J.R. et LAmiroy, B. (1994), Expressions figées de Belgique, Linguisticae Investigationes, XVIII, 2, pp. 285-321.

KLEIN, J.R. et LAMiroy, B. (1995), Les expressions figées du français de Belgique, in LABELLE, J. \& LECLÈre, C. (éds), Lexiques-grammaires comparés en français. Amsterdam : Benjamins, pp. 37-52.

KLEIN, J.R. et LAMIROY, B. à paraitre. Relations systématiques entre expressions figées à travers quatre variétés du français, in Bolly, C., Klein, J. \& Lamiroy, B. (éds), La Phraséologie dans tous ses états. Cabiers de l'Institut de Linguistique de Louvain (numéro spécial), sous presse. 
KLEIN, J.R. et Rossari, C. (2003), Figement et variations en français de Belgique, de France, du Québec et de Suisse, Linguisticae Investigationes, 26-2, pp. 203-214.

Lamiroy, B. (éd.) (1998), Le lexique-grammaire. Travaux de Linguistique, 37, numéro spécial.

LAmiroy, B. (2003), Les notions linguistiques de figement et de contrainte, Linguisticae Investigationes, 26, 1, pp. 1-14.

LAmiroy, B. à paraitre. Le français de Belgique et les locutions verbales figées, in Delcourt F. (éd.), Hommages à André Goosse. Revue belge de philologie et d'bistoire, numéro spécial (sous presse).

LAMiroy, B. \& LECLÈre, C. (2003), Expressions verbales figées et variation en français, in Clas, A. et al. (éds), L'éloge de la différence : la voix de l'Autre. Paris: AUF, Coll. Actualités scientifiques, pp. 209-225.

Lamiroy, B., Klein, J.R., LAbelle, J. \& Leclère, C. (2003), Expressions verbales figées et variation en français : le projet BFQS. Cabiers de lexicologie 2, pp. 153-172.

LAMiroy, B. \& KLEIN, J.R. (2004), La structure de la phrase simple en français de Belgique, in Laporte, E., Leclère, C., Piot, M. \& Silberztein, M. (éds), Syntaxe, lexique et lexiquegrammaire. Volume dédié à Maurice Gross. Amsterdam : Benjamins, pp. 343-373.

Martin, R. (1997), Sur les facteurs du figement lexical, in Martins-Baltar, M. (éd.), pp. 291-305.

Martins-Baltar, M. (éd) (1997), La locution entre langue et usages. Paris: ENS éditions. Fontenay-St Cloud, 3 vol.

Massion, F. (1987), Dictionnaire de belgicismes. Berne : P. Lang.

Meney, L. (1999), Dictionnaire Québecois-Français. Montréal : Guérin.

MejRI, S. (1997), Le figement lexical. Tunis : Publications de la faculté des lettres de la Manouba.

MEJRI, S. (éd.) (2003), Le figement lexical. Cahiers de lexicologie, 82,1.

MisRI, G. (1987), Le figement linguistique en français contemporain. Paris V, Université René Descartes, thèse de doctorat.

Moon, R. (1998), Fixed Expressions and idioms in English, a corpus-based approach. Oxford: Clarendon Press.

Nunberg, G., SAG, I., WASOW, T. (1994), Idioms, Language 70, pp. 491-538.

RÉZEAU, P. (2001), Dictionnaire des régionalismes de France. Bruxelles : De Boeck-Duculot.

SCHAPIRA, C. (1999), Les stéréotypes en français : proverbes et autres formules. Paris : Ophrys.

SENELLART, J. (1998), Reconnaissance automatique des entrées du lexique-grammaire des phrases figées, in LAMiroy, B. (éd), pp. 109-127.

Svensson, M.H. (2004), Critères de figement. L'identification des expressions figées en français contemporain. Umeå, Umeå : University.

Thibaut, A. \& KNECHT, P. (1997), Dictionnaire suisse romand: particularités lexicales du français contemporain. Genève : Zoé. 
Béatrice Lamiroy et Jean René Klein

TOLLIS, F. (éd.) (2001), La locution et la périphrase. Du lexique à la grammaire. Paris : L'Harmattan.

TOMASELlo, M (éd.) (1998), The new psychology of language. Cognitive and Functional Approaches to Language Structure. Mahwah, NJ : LEA.

Wray, A. (2002), Formulaic Language and the Lexicon. Cambridge : Cambridge University Press. 